DSF $-29 / 2007$

\title{
AN UNKNOWN STORY: MAJORANA AND THE PAULI-WEISSKOPF SCALAR ELECTRODYNAMICS
}

\author{
S. ESPOSITO
}

\begin{abstract}
An account is given of an interesting but unknown theory by Majorana regarding scalar quantum electrodynamics, elaborated several years before the known Pauli-Weisskopf theory. Theoretical calculations and their interpretation are given in detail, together with a general historical discussion of the main steps towards the building of a quantum field theory for electrodynamics. A possible peculiar application to nuclear constitution, as conceived around 1930, considered by Majorana is as well discussed.
\end{abstract}

\section{The Known story of early Quantum Electrodynamics}

Until a few years ago it had been impossible to construct a theory of radiation which could account satisfactorily both for interference phenomena and the phenomena of emission and absorption of light by matter. The first set of phenomena was interpreted by the wave theory, and the second set by the theory of light quanta. It was not until in 1927 that Dirac succeeded in constructing a quantum theory of radiation which could explain in an unified way both types of phenomena [1].

1.1. First steps in Quantum Field Theory. With these words, according to what was later published [1], Enrico Fermi opened the first of his renowned lectures on the "Quantum theory of radiation", delivered at the University of Michigan - Ann Arbor in 1930. The basic problem for the building up of a quantum electrodynamics was, in fact, the well recognized dichotomy concerning the description of particles (electrons) in a wave-mechanical way, as well as the description of light quanta (photons) in the framework of the Maxwell theory of electromagnetism.

The first preliminary step towards the clarification of such an issue, just after the appearance of Quantum Mechanics, was carried out by Max Born, Werner Heisenberg and Pascual Jordan in 1926 [3]. By studying the problem of the vibrations of a one-dimensional string fixed at both sides, Jordan interpreted the quantum numbers of individual oscillators as the number of light quanta with given frequency in each cell (in the Bose-Einstein sense). This one-to-one correspondence proved to give the correct fluctuation law for cavity radiation, thus starting to shed some light in the puzzle mentioned earlier. The method developed by Jordan was then applied to a general theory of electromagnetic radiation by Paul A.M. Dirac in the fundamental papers $[4,5]$ quoted above by Fermi.

The key viewpoint in the Dirac theory of radiation is to consider a light emitting or absorbing atom and the radiation in the space [around it] as a single system. This is described, apart from the 
atomic coordinates, in terms also of some other variables which specify the values of the potentials of the electromagnetic field in any point of the space. If, in order to simplify the discussion, we think at the radiation inside a cavity, we know that the radiation [field] may vibrate according to certain characteristic frequencies, depending on the form and dimension of the cavity. In this case, for each fundamental vibration, one can take the value of the electric field at a given time in one of the antinodes (or a quantity proportional to it) as the coordinate of the radiation field, so that we have one coordinate for each fundamental frequency. If we allow the walls of the cavity to tend to the infinity, the fundamental vibrations of the radiation tend to given asymptotic distributions in such a way that, in the limiting case, any influence of the walls of the cavity on the phenomenon ceases, and we are led to the case of the free space. [6]

Dirac described the system at hand in hamiltonian terms, and the wavefunction of the perturbed hamiltonian system, satisfying the time-dependent Schrödinger equation, was expanded in terms of the unperturbed wavefunctions (plane waves). Here the main point is that the expansion coefficients $a_{r}, a_{s}^{*}$ (or some other coefficients $b_{r}, b_{s}^{*}$ related to $a_{r}, a_{s}^{*}$ by a given transformation), taken as canonical conjugate variables, are assumed to be q-numbers satisfying the commutation relations

$$
\left[a_{r}, a_{s}^{*}\right]=\delta_{r, s}, \quad\left[a_{r}, a_{s}\right]=\left[a_{r}^{*}, a_{s}^{*}\right]=0 .
$$

This further or "second" quantization of such operators proved useful in demonstrating that the Hamiltonian considered by Dirac was for an assembly of particles obeying the Bose-Einstein statistics. The hamiltonian interaction term between the radiation field and the atom was shown to have the same form of that pertaining to an assembly of light quanta interacting with the atom. "There is thus a complete formal reconciliation between the wave and light quantum points of vie" [5]. The Dirac theory was soon recognized to describe correctly and satisfactorily the emission and absorption of light, the dispersion phenomena and, more in general, all the phenomena related to energy exchange between light and matter.

Few months later the appearance of the Dirac papers, Jordan and Oskar Klein [7] extended the Dirac theory to the case of a system of massive bosons and, finally, Jordan and Eugene P. Wigner in 1928 [8] obtained a quantization method for fermions. They showed that, for electrons, the field cannot be expanded in terms of operators satisfying the commutation relations in Eq. (1), and introduced anticommutators for the field variables in order to take into account the Pauli exclusion principle. The use of canonical commutation or anticommutation relations for the operators $a, a^{*}$ raised the problem of Lorentz invariance of the second-quantized theory. For the charge-free case, the problem of a relativistically invariant formulation of the Dirac theory ${ }^{1}$ was considered by Jordan and Wolfgang Pauli later in 1928 [9] (addressing, in addition, also the problem with the infinite zero-point energy) who, in a certain sense, generalized the Dirac method by quantizing directly the field strengths instead of the potentials. As Dirac, they decomposed the radiation

\footnotetext{
${ }^{1}$ In the Dirac theory, the electromagnetic potential was separated into a radiation field $\boldsymbol{A}$ and a static Coulomb potential $\varphi$ in a way that gauge and Lorentz invariance was not preserved, on the contrary to what manifestly happened in the classical electrodynamics theory.
} 
field in its harmonic components, and quantization conditions similar to those for the harmonic oscillator were applied to each of these components. The commutation relations between the electric and magnetic field components were derived in such a way that relativistic invariance holds, showing that, inter alia, the quantized electromagnetic field propagates at the velocity of light and that electric and magnetic fields may be measured to arbitrary accuracy at two points with a space-time separation.

1.2. Towards a Quantum Field Theory for Electrodynamics. The formalism introduced by Jordan and Pauli was quite general, but it applied only to the charge-free case, whilst Dirac considered, though in an approximate way, also the presence of electric charges. However, as explicitly stated, the Dirac expansion in unperturbed plane waves assumed the electromagnetic field to be only a radiation field, so that the results applied only far from the charges generating the field. Thus the need for a further generalization, taking into account, for example, the action between two neighbouring atoms or two electrons in the same atom, was felt by many authors. In 1929 Heisenberg and Pauli [10, 11], on one hand, and Fermi $[12,6,13,1]$ on the other hand studied and solved in different ways this problem. The key point was just to recognize that "the Hamiltonian representing the natural quantum translation of the classical electrodynamics, in the sense given by the principle of correspondence, is simply obtained by adding to the Hamiltonian of the Dirac radiation theory a term representing the electrostatic energy of the electric charges" [13]. The two approaches were quite different in the formalism used.

A general quantum theory of the electromagnetic field was constructed by Heisenberg and Pauli by a method in which the values of the electromagnetic potentials an all the points of space are considered as variables.

Independently [Fermi] proposed another method of quantization of the electromagnetic field starting from a Fourier analysis of the potentials. though Heisenberg and Pauli method puts in evidence much more clearly the properties of relativistic invariance and is in many regards more general, [...] [Fermi method] is more simple and more analogous to the methods used in the theory of radiation. [1].

The method adopted by Heisenberg and Pauli was, in fact, very general and based on lagrangian field theory. After the writing of a Lagrangian density $L$ for the fields $\varphi_{a}$ describing a given physical system, and the calculation of the canonical momenta $\Pi_{a}$ conjugate to the fields $\varphi_{a}$ and the Hamiltonian density $H$ of the system, the quantization is introduced by requiring that $\varphi_{a}$ and $\Pi_{a}$ be q-numbers satisfying the relations

$$
\left[\Pi_{a}, \varphi_{a}\right]=\frac{\hbar}{i}
$$

where the commutators (for bosons) or anticommutators (for fermions) are evaluated at equal times for different spatial points. This general formalism was applied to the cases of a Dirac field and of the electromagnetic field, by studying the fundamental invariance and conservation laws. However, a major difficulty in applying such a formalism to the electromagnetic field was that the canonical momentum conjugate to the scalar potential is zero so that, for this field component, Eq. (2) cannot apply, the left-hand-side being identically zero. A first attempt to solve this 
problem [10] was to introduce a Lagrange multiplier in the original Lagrangian density, preserving gauge and relativistic invariance, this parameter being set to zero at the end of any calculation. Subsequently Heisenberg and Pauli demonstrated that, in a relativistically invariant manner, one can always choose a gauge where the scalar potential is zero, $\varphi=0$, so that calculations can be carried out in the Coulomb gauge without additional terms.

1.3. The problem with the negative energy states. In the just raising quantum electrodynamics, the matter fields, describing e.g. electrons, were of course assumed to be bispinors to which the successful Dirac theory of spin 1/2 particles applied [14]. This brought to the quantum electrodynamics theory, in addition to the known problem of an infinite self-energy term for the electron, the puzzle which seemed intrinsically associated with any relativistic theory (and the Dirac theory of electrons in particular), i.e. that of the negative energy states and the possible transitions from positive to negative energy states. Heisenberg and Pauli simply discarded such a problem, regarding the negative energy states as an "inconsistency of the theory [...], which must be accepted as long as the Dirac difficulty is unexplained" [10]. On the other hand, also Fermi recognized that this ...

... theory has two fundamental defects that, rather than of electrodynamic origin, may be considered as coming from the incomplete knowledge of the electron structure. They are the possibility that the Dirac electron has to jump to negative energy levels and the fact that the self-energy takes an infinite value by assuming an exactly pointlike electron [13].

For our purposes, we definitely do not discuss further about the last point. Instead, regarding the problem with the negative energy states, it was soon well clear to Dirac himself that from his equation it follows that a negative energy electron moves in an external field as if it had positive charge and energy. This original observation led Hermann Weyl in 1929 to "expect that, among the two pairs of components of the Dirac [wavefunction] one pair corresponds to the electron, while the other to the proton" [15]. This association was further considered (for some time) by Dirac in the framework of his "hole theory", with the identification of "the holes in the distribution of negative energy electrons" with the protons [16], although novel difficulties arose with such interpretation. Here we only quote from the Dirac paper appeared at the very beginning of 1930 :

Can the present theory account for the great dissymmetry between electrons and protons, which manifest itself through their different masses and the power of protons to combine to form heavier atomic nuclei? It is evident that the theory gives, to a large extent, symmetry between electrons and protons [...] The symmetry is not, however, mathematically perfect when one takes interaction between the electrons into account. [...] The consequences of this dissymmetry are not very easy to calculate on relativistic lines, but we may hope it will lead eventually to an explanation of the different masses of proton and electron [16].

As well known, such difficulty in the interpretation of the theory, and some others related to it (i.e., the too high rate of annihilation of electrons and protons), were 
later overcome by Dirac himself, after the Weyl proof that holes necessarily represented particles with the same mass as an electron [17]. "A hole, if there were one, would be a new kind of particle, unknown to experimental physics, having the same mass and opposite charge to an electron. We may call such a particle an anti-electron" [18]. This novel interpretation, though highly controversial, was eventually confirmed by the cosmic-rays experiments by Carl D. Anderson [19] in 1932. However, the fact that the observed positive electrons were indeed Dirac antiparticles was not clear for some time, but was fully recognized only after the appearance of the experimental results on cosmic-rays showers by Patrick M. Blackett and Giuseppe P.S. Occhialini [20], followed by a discussion of their results within the framework of the Dirac theory. Notwithstanding this, especially Pauli was very critical with the Dirac theory of negative energy states. "I do not believe in your perception of 'holes', even if the existence of the 'antielectron' is proved", wrote Pauli to Dirac on May 1, 1933 [21]. And, more specifically, in a letter to Heisenberg of July 24, 1933, Pauli pointed out that the ...

... play with infinite concepts in the present framework is unacceptable and will eventually lead to contradictions, In particular, I don't see how the Coulomb interaction energy can be eliminated unequivocally and free from arbitrariness with the infinitely many occupied states [21].

An attempt was made by Heisenberg (in a letter to Pauli of July 17, 1933 [21]) to fold hole theory into the Heisenberg-Pauli formalism of quantum electrodynamics, in order to try to take into some account Pauli criticism. But the most important step in this direction was carried out by Vladimir Fock, Wendell Furry and Julius R. Oppenheimer in 1934. Inspired by the Dirac idea to consider an annihilation as a lack of one electron with negative energy, they defined the creation and annihilation operators $b^{*}, b$ of the antielectrons as the corresponding operators of annihilation and creation $a, a^{*}$ of the electrons with positive energy. The Dirac field $\psi$ may then be expanded as two sums over normal modes with positive and negative energies, in such a way that the energy of the system,

$$
H=\sum_{k}^{E_{k}>0} E_{k} a_{k}^{*} a_{k}+\sum_{k}^{E_{k}<0}\left|E_{k}\right| b_{k}^{*} b_{k}+E_{0},
$$

when the (infinite) vacuum energy $E_{0}$ is subtracted, is the sum of two terms that are positive definite.

1.4. Quantization of the Klein-Gordon equation. The problem of the negative energy states was solved, for a charge particle with spin 0, in 1934 by Pauli and Victor F. Weisskopf [22]. At the time their work was viewed just as a theoretical exercise (the authors themselves considered their theory to be truly a "curiosity", thus choosing to publish it in a not first-line journal [23]), since no "elementary" spin 0 particle ${ }^{2}$ was then known. Nevertheless, as we will see below, it proved to be a very important step in the development of the quantum theory of electrodynamics.

The Pauli-Weisskopf paper "consistently applies the Heisenberg-Pauli formalism of the quantization of wave fields to the scalar relativistic wave-equation for matter

\footnotetext{
${ }^{2}$ Of course, spin 0 particles were known since 1920s, as for example the hydrogen atom in its ground state or the helium nucleus. However neither of these particles were "elementary", so that no need was felt to describe them by means of a relativistic wave-equation.
} 
fields when the particles have Bose-Einstein statistics" [22]. The scalar relativistic wave-equation was that earlier discovered by Schrödinger and, independently, by Klein and Walter Gordon (for a discussion see e.g. [23]):

$$
\left[\frac{\hbar^{2}}{c^{2}} \frac{\partial^{2}}{\partial t^{2}}-\hbar^{2} \nabla^{2}+m^{2} c^{2}\right] \psi=0 .
$$

The original rejection by Schrödinger (and others) of this equation was due to its incorrect predictions, with respect to the experimental observations, about the fine structure of the spectrum of the hydrogen atom. This was correctly ascribed to the fact that such an equation did not take into account the electron spin. However the later appearance of the Dirac theory for the spinning electron, which gave correct experimental predictions, shifted the attention to the problem of non positive definite probabilities, found in the Schrödinger-Klein-Gordon theory but not in the Dirac theory. This brought to the generally-accepted assumption about the non-existence of spin 0 (elementary) particles, which was reinforced by the interpretation underlying the hole theory. In fact, the exclusion principle that played a key role in that interpretation could not be applied to scalar particles, so that no mechanism existed that prevented the particles to pass from positive energy states to negative ones. It is a merit of the Pauli-Weisskopf theory to have shown that non intrinsically wrong arguments lie in the Schrödinger-Klein-Gordon theory that justifies the derivation of the Dirac theory; they just apply to particles with different spin, and the interpretation of antiparticles in terms of holes is no longer necessary.

As announced in the abstract of their paper, Pauli and Weisskopf adopted the canonical formalism introduced earlier by Heisenberg and Pauli, and applied it first of all to the quantization of the wave field in the force-free case. By starting from the Lagrangian density for the complex scalar field $\psi$ and its conjugate $\psi^{*}$, they deduced the momentum $\Pi$ and $\Pi^{*}$, which are canonically conjugate to $\psi$ and $\psi^{*}$, and imposed the commutation relations (2), appropriate for the Bose-Einstein statistics. The expressions for the Hamiltonian $H$, charge density $\rho$ and current density $s$ operators were obtained and, by decomposing the fields into hermitian parts,

$$
\psi=\frac{1}{\sqrt{2}}\left(u_{1}+i u_{2}\right), \quad \Pi=\frac{1}{\sqrt{2}}\left(p_{1}-i p_{2}\right)
$$

(and similarly for $\psi^{*}$ and $\Pi^{*}$ ), they showed that the charge situated within an arbitrary finite region $V$

$$
\int_{V} \rho \mathrm{d} V=\int_{V}\left(p_{1} u_{2}-p_{2} u_{1}\right) \mathrm{d} V
$$

measured in units of electron charge $e$, has the eigenvalues $0, \pm 1, \pm 2, \ldots, \pm N$. Then Pauli and Weisskopf considered a complete system of orthonormalized eigenfunctions (plane waves) in the momentum space, and expanded the field functions in terms of these:

$$
\begin{aligned}
\psi & =\frac{1}{V^{1 / 2}} \sum_{k} q_{k} \mathrm{e}^{i \boldsymbol{k} \cdot \boldsymbol{x}}, \\
\Pi & =\frac{1}{V^{1 / 2}} \sum_{k} p_{k} \mathrm{e}^{-i \boldsymbol{k} \cdot \boldsymbol{x}}
\end{aligned}
$$


(and similarly for $\psi^{*}$ and $\Pi^{*}$ ). The (non-hermitian) q-numbers $q_{k}, p_{k}, q_{k}^{*}, p_{k}^{*}$, or the annihilation and creation operators $a_{k}, b_{k}, a_{k}^{*}, b_{k}^{*}$ derived by them, satisfy canonical commutation relations, and these were used to write the total energy $H$ and charge $Q$ (and momentum and current) as follows:

$$
\begin{gathered}
H=\sum_{k}\left(p_{k}^{*} p_{k}+E_{k}^{2} q_{k}^{*} q_{k}\right)=\sum_{k} E_{k}\left(a_{k}^{*} a_{k}+b_{k}^{*} b_{k}+1\right), \\
Q=-i \sum_{k}\left(p_{k} q_{k}-p_{k}^{*} q_{k}^{*}\right)=\sum_{k}\left(a_{k}^{*} a_{k}-b_{k}^{*} b_{k}\right) .
\end{gathered}
$$

Here $E_{k}=+c \sqrt{\hbar^{2} k^{2}+m^{2} c^{2}}$ is always positive, and the same applies to the total energy $H$ regardless of the zero-point energy (the term corresponding to +1 in Eq. (8)), this having a "decisive physical meaning". Furthermore, it was shown that the operators $N_{k}^{+}=a_{k}^{*} a_{k}$ and $N_{k}^{-}=b_{k}^{*} b_{k}$, appearing above, have non-negative integer eigenvalues: " $N_{k}^{+}$signifies the number of particles with charge number +1 and momentum $\hbar \boldsymbol{k}$, and $N_{k}^{-}$signifies the number of particles with charge number -1 and momentum $-\hbar \boldsymbol{k}$ ". The existence of two different kinds of operators $a, b$ playing the same role in the Hamiltonian, made evident the fact that the PauliWeisskopf theory is a quantum theory for two scalar particles with the same mass, these being interpreted as the particle and the corresponding antiparticle.

The next step was to consider the interaction of the scalar particle with an electromagnetic field, described by the potentials $A_{\mu}$. This was achieved simply by the replacement

$$
\frac{\partial \psi}{\partial x^{\mu}} \longrightarrow \frac{\partial \psi}{\partial x^{\mu}}-\frac{i e}{\hbar c} A_{\mu} \psi
$$

(and similarly for $\psi^{*}$ ) in the original Lagrangian function, and adding to this the appropriate kinetic term for the electromagnetic field. The canonical formalism was then applied to the present case, and the important result was obtained that "the eigenvalues of the [novel operator for the] charge density remain the same as in the force-free case, even when external potentials are present", so that the previous basic interpretation of the theory remained unchanged. The complete Hamiltonian function was divided into three terms, pertaining to the matter fields, the radiation field and the interaction between them, respectively. This was later exploited in order to calculate the relevant effects, such as pair annihilation and creation by light quanta (and the polarization of the vacuum). "The frequency of these processes proves to be of the same order of magnitude as the frequency for particles of the same charge and mass, which follows from the Dirac hole theory".

Our account of the known story of quantum electrodynamics stops here. This is certainly not due to the lack of further cornerstones, which came out especially after the Second World War, but because the further developments simply focused on (very important) formal improvements of the theory and their physical interpretation (recall also that, in our discussion, we have completely disregarded the problems with the infinities). With the Pauli-Weisskopf theory a key point has been reached, with the proof of the most important fact that antiparticles were not just a peculiarity of Dirac theory for spin $1 / 2$ fermions, but also a feature of a quantum field theory for charged bosons. As well important was that the Pauli-Weisskopf paper corrected the generally-accepted view that the inclusion of the principles of the theory of special relativity into quantum mechanics required necessarily a spin $1 / 2$, 
while it showed that scalar quantum electrodynamics was able to predict physical processes similar to spin $1 / 2$ quantum electrodynamics.

\section{An unknown contribution by Ettore Majorana}

According to Edoardo Amaldi, during the winter of 1928-29 Fermi started studying the quantum theory of radiation. [...] His formulation formed the subject of a course given in April 1929 at the Institut Poincaré in Paris and in a more complete form at the Summer School of Theoretical Physics at Ann Arbor, Michigan during the summer of 1930. [...] While doing this work Fermi taught his result to several of his pupils and friends including Amaldi, Majorana, Racah, Rasetti and Segrè. Every day when work was over he gathered the various people mentioned above around his table and started to elaborate before them, first the basic formulation of quantum electrodynamics and then, one after the other, a long series of applications of the general principles to particular physical problems [24].

The study of such a subject particularly impressed, among the others, Ettore Majorana, this being widely recorded in his personal study notes, the "Volumetti" [25], and especially in his research notes, the "Quaderni" [26]. These treasures of theoretical physics, conserved at the Domus Galilaeana in Pisa (Italy), have been made available to a general audience only recently with the mentioned publications, and are bright confirmations of his high talent as theoretical physicist, as acknowledged in several occasions by Fermi, Heisenberg and many others (see, for example, Ref. $[27])$.

Here we concentrate only on some interesting results obtained by Majorana several years before the appearance of the Pauli-Weisskopf paper. The original material discussed below is contained in the Quaderno No. 2, which is not explicitly dated. However an accurate inspection of the arguments present in it and the comparison with the existing literature (as, for example, the Franco Rasetti's important work on the Raman spectra of molecules [28]) and with the Volumetto III (which is dated 1929) allows to conclude that such work was carried out at the end of 1929 or early in 1930. This fits well with the independent piece of information by Amaldi about the Fermi private course on quantum electrodynamics (and with another news reported below).

Before entering into the details of the theory of Majorana, we disclose the results achieved, by reporting what seems the only testimony on these results (Majorana never published them), as recalled by Gian Carlo Wick. ${ }^{3}$

[...] I was admitted as a bystander to an international meeting on Nuclear Physics, which was organized ny the Royal Academy in Rome in the fall of 1931. [...]

I was asked by Heitler to act as a sort of interpreter between him and Majorana. He spoke hardly any Italian, and Majorana's German was a bit weak. So during lunches, Heitler expressed a

\footnotetext{
${ }^{3}$ Wick talked several times about the following episode (see, for example, [27], [29]); in the following we report an excerpt from Ref. [29] and an handwritten note by Wick conserved at the Wick Archive of the Scuola Normale Superiore in Pisa. This last paper contains many interesting details, lacking in others testimonies.
} 
curiosity in what Majorana was doing; Fermi must have told him how bright he was. So Majorana began telling, in that detached and somewhat ironical tone, which was typical of him, especially when discussing his own work, that he was developing a relativistic theory for charged particles. It was not true, he said, that the Schrödinger equation for a relativistic particle had to have the form indicated by Dirac. It was clear by $\mathrm{n}$ ow, that in a relativistic theory one had to start from a field theory and for this purpose the Klein-Gordon wave-equation was just as legitimate as Dirac's. If one took that, and quantized it, one got a theory with consequences quite similar to Dirac positron theory, with positive and negative charges, the possibility of pair creation etc. You can see what I am driving at: Majorana had the Pauli-Weisskopf scheme all worked out already at that time. Please do not think I am disputing the merit of these authors. Majorana never published this work, he did not seem to take it very seriously, and I don't think Pauli and Weisskopf ever even heard of it. Heitler probably forgot all about it, and so did I, until I saw the paper by Pauli and Weisskopf.

I must confess that I knew enough to understand the gist of what he was saying, but not enough to appreciate how novel and original it was. Heitler probably did, because his comment, as I recall it, was: 'I hope you will publish this'. [...] [But] in the case I have described publication never occurred .

2.1. Majorana theory - first part. In his notebooks, Majorana started to consider scalar quantum electrodynamics with two preliminary studies on the quantization of the free (without interaction with the electromagnetic field) Klein-Gordon equation and on the quantization of the free electromagnetic field. Here we do not go in any detail about these preliminary studies (the complete treatment of the problem may be found in Sect.s 2.7, 2.8 and 7.6 of Ref. [26]), but immediately report on the first theoretical framework considered by Majorana.

The starting point was the following variational principle for a complex scalar field $\psi$ and its complex conjugate $\bar{\psi}$ interacting with the electromagnetic potentials $\varphi, \boldsymbol{A}$ :

$$
\begin{aligned}
& \delta \int\left\{\frac { h ^ { 2 } } { 8 \pi ^ { 2 } m } \left[\frac{1}{c^{2}}\left(\frac{\partial}{\partial t}+\frac{2 \pi i}{h} e \varphi\right) \bar{\psi}\left(\frac{\partial}{\partial t}-\frac{2 \pi i}{h} e \varphi\right) \psi\right.\right. \\
&\left.\left.-\left(\boldsymbol{\nabla}-\frac{2 \pi i}{h c} e \boldsymbol{A}\right) \bar{\psi} \cdot\left(\boldsymbol{\nabla}+\frac{2 \pi i}{h c} e \boldsymbol{A}\right) \psi\right]-\frac{1}{2} m c^{2} \bar{\psi} \psi\right\} \mathrm{d} \tau=0 .
\end{aligned}
$$

Here $-e, m$ are the electric charge and mass of the field $\psi$, respectively, while $\mathrm{d} \tau=\mathrm{d} t \mathrm{~d} V$ is the 4 -volume element. By taking variations with respect to $\psi, \bar{\psi}$ one obtains the wave-equations:

$$
\begin{aligned}
& {\left[\frac{h^{2}}{8 \pi^{2} m c^{2}}\left(\frac{\partial}{\partial t}-\frac{2 \pi i}{h} e \varphi\right)^{2}-\frac{h^{2}}{8 \pi^{2} m}\left(\boldsymbol{\nabla}+\frac{2 \pi i}{h c} e \boldsymbol{A}\right)^{2}+\frac{1}{2} m c^{2}\right] \psi=0,} \\
& {\left[\frac{h^{2}}{8 \pi^{2} m c^{2}}\left(\frac{\partial}{\partial t}+\frac{2 \pi i}{h} e \varphi\right)^{2}-\frac{h^{2}}{8 \pi^{2} m}\left(\nabla-\frac{2 \pi i}{h c} e \boldsymbol{A}\right)^{2}+\frac{1}{2} m c^{2}\right] \bar{\psi}=0 .}
\end{aligned}
$$


The field $P$ canonically conjugate to $\psi$ is

$$
P=\frac{h^{2}}{8 \pi^{2} m c^{2}}\left(\frac{\partial}{\partial t}+\frac{2 \pi i}{h} e \varphi\right) \bar{\psi}
$$

and similarly for $\bar{P}$. The Hamiltonian of the system considered was obtained to be:

$$
\begin{aligned}
H= & \int\left\{\frac{h^{2}}{8 \pi^{2} m c^{2}} \frac{\partial \bar{\psi}}{\partial t} \frac{\partial \psi}{\partial t}+\frac{1}{2} m c^{2} \bar{\psi} \psi+\frac{h^{2}}{8 \pi^{2} m} \nabla \bar{\psi} \cdot \nabla \psi\right. \\
& +\frac{h e}{4 \pi i m c}\left[\bar{\psi} \boldsymbol{A} \cdot \boldsymbol{\nabla} \psi-\psi \boldsymbol{A} \cdot \boldsymbol{\nabla} \bar{\psi}+\frac{e^{2}}{2 m c^{2}}\left(A^{2}-\varphi^{2}\right) \bar{\psi} \psi\right\} \mathrm{d} V \\
= & \int\left\{\frac{8 \pi^{2} m c^{2}}{h^{2}} \bar{P} P+\frac{2 \pi i}{h} e \varphi(\psi P-\bar{\psi} \bar{P})+\frac{1}{2} m c^{2} \bar{\psi} \psi+\frac{h^{2}}{8 \pi^{2} m} \boldsymbol{\nabla} \bar{\psi} \cdot \boldsymbol{\nabla} \psi\right. \\
& \left.+\frac{h e}{4 \pi i m c}[\bar{\psi} \boldsymbol{A} \cdot \boldsymbol{\nabla} \psi-\psi \boldsymbol{A} \cdot \boldsymbol{\nabla} \bar{\psi}]+\frac{e^{2}}{2 m c^{2}}|A|^{2} \bar{\psi} \psi\right\} \mathrm{d} V
\end{aligned}
$$

while the total number $N$ of particles is given by

$$
N=\int-\frac{2 \pi i}{h}(\psi P-\bar{\psi} \bar{P}) \mathrm{d} V
$$

Quantization was achieved by imposing the following conditions (with appropriate interpretation of the symbols; we prefer to adhere to the original notation, not intended for publication, as long as the meaning is sufficiently clear):

$$
[\psi, P]=1, \quad[\bar{\psi}, \bar{P}]=1
$$

(while the other commutators vanish). At this point Majorana introduced the transformations:

$$
\psi=\frac{\psi_{0}-i \psi_{1}}{\sqrt{2}}, \quad \psi=\frac{P_{0}+i P_{1}}{\sqrt{2}},
$$

and similarly for $\bar{\psi}, \bar{P}$, so that the quantization conditions change as follows:

$$
\begin{gathered}
{\left[\psi_{0}, P_{0}\right]=1, \quad\left[\psi_{1}, P_{1}\right]=1,} \\
{\left[\psi_{0}, P_{1}\right]=\left[\psi_{0}, \psi_{1}\right]=\left[P_{0}, P_{1}\right]=\left[\psi_{1}, P_{0}\right]=0 .}
\end{gathered}
$$

The novel fields are then expanded in plane waves:

$$
\begin{array}{ll}
\psi_{0}=\sum_{r} q_{0}^{r} u^{r}, & \psi_{1}=\sum_{r} q_{1}^{r} u^{r}, \\
P_{0}=\sum_{r} p_{0}^{r} u_{r}, & P_{1}=\sum_{r} p_{1}^{r} u_{r},
\end{array}
$$

where $q_{0}, q_{1}, p_{0}, p_{1}$ are four hermitian operators, and the functions $u_{r}$ satisfy:

$$
\nabla^{2} u_{r}+k_{r}^{2} u_{r}=0, \quad \quad \int u_{r}^{2} \mathrm{~d} V=1 .
$$

From now on (for this first part of the Majorana theory), the discussion was specialized to consider only the case of scalar fields without interaction with the electromagnetic field. The Hamiltonian takes then the simpler form (here and below, 
for simplicity, we suppress the indices $r$ if no confusion may be generated):

$$
\begin{aligned}
H_{0}= & \sum\left\{\frac{4 \pi^{2} m c^{2}}{h^{2}} p_{0}^{2}+\left(\frac{h^{2} k^{2}}{16 \pi^{2} m}+\frac{1}{4} m c^{2}\right) q_{0}^{2}\right. \\
& \left.+\frac{4 \pi^{2} m c^{2}}{h^{2}} p_{1}^{2}+\left(\frac{h^{2} k^{2}}{16 \pi^{2} m}+\frac{1}{4} m c^{2}\right) q_{1}^{2}\right\} .
\end{aligned}
$$

By introducing the quantity $\nu$ defined by

$$
\nu^{2}=\frac{m^{2} c^{4}}{h^{2}}+\frac{c^{2} k^{2}}{4 \pi^{2}}
$$

the total energy is then:

$$
E=\sum_{r} N_{r} c \sqrt{m^{2} c^{2}+p_{r}^{2}}=\sum N_{r} h \nu_{r}=\sum N_{r} E_{r}
$$

where the total number of particles $N$, and the total charge $Z$ could be written as:

$$
N=\sum_{r} N_{r}, \quad Z=\sum_{r} Z_{r}
$$

Here the operator $N_{r}$ has eigenvalues $0,1,2, \ldots$ (see below) while $Z_{r}$ takes the values $N_{r}, N_{r}-2, N_{r}-4, \ldots,-N_{r}$; from $\left|Z_{r}\right| \leq N_{r}$, Majorana then pointed out that in his theory $|Z| \leq N$.

It is worthwhile to stress the fact that Majorana thus succeeded to decompose the charged scalar field into a sum of normal modes, each having a relativistic energy $c \sqrt{m^{2} c^{2}+p_{r}^{2}}$.

A short insert is introduced at this stage, where the electrostatic interaction through the potentials $\boldsymbol{A}=0, \varphi \neq 0$ is dealt with. The scalar potential is expanded as:

$$
\varphi=\sum_{r} \varphi_{r} u_{r}, \quad \varphi_{r}=\int \varphi u_{r}^{2} \mathrm{~d} V
$$

and, by defining

$$
\varphi_{r s}=\int \varphi u_{r} u_{s} \mathrm{~d} V
$$

the complete Hamiltonian is given by:

$$
H=H_{0}-\frac{2 \pi}{h} \sum_{r, s} \varphi_{r s}\left(q_{0}^{r} p_{1}^{s}-q_{1}^{r} p_{0}^{s}\right) .
$$

Turning back to the main problem, the calculations were now simplified by taking units where $h=2 \pi$ and $\nu=1 / 2 \pi$, so that $h \nu=1$. In these units, the operators $N, Z$ were given by:

$$
\begin{gathered}
N=\frac{1}{2} P_{0}^{2}+\frac{1}{2} Q_{0}^{2}+\frac{1}{2} P_{1}^{2}+\frac{1}{2} Q_{1}^{2}-1, \\
Z=Q_{0} P_{1}-Q_{1} P_{0}
\end{gathered}
$$

(with obvious meaning of the changed notations). Explicit calculations of the matrix elements of the operators $Q_{0}, Q_{1}, P_{0}, P_{1}, N, Z$ are carried out, in the chosen basis of eigenfunctions, by starting from the following complete set of commutation relations:

$$
\begin{gathered}
P_{0} Q_{0}-Q_{0} P_{0}=\frac{1}{i}, \quad P_{1} Q_{1}-Q_{1} P_{1}=\frac{1}{i}, \\
P_{0} P_{1}-P_{1} P_{0}=\cdots=0
\end{gathered}
$$




$$
\begin{array}{ll}
N P_{0}-P_{0} N=i Q_{0}, & -\left(Z P_{0}-P_{0} Z\right)=-i P_{1}, \\
N Q_{0}-Q_{0} N=-i P_{0}, & -\left(Z Q_{0}-Q_{0} Z\right)=-i Q_{1}, \\
N P_{1}-P_{1} N=i Q_{1}, & -\left(Z P_{1}-P_{1} Z\right)=i P_{0}, \\
N Q_{1}-Q_{1} N=-i P_{1}, & -\left(Z Q_{1}-Q_{1} Z\right)=i Q_{0} .
\end{array}
$$

Here we report only the explicit expressions for the total number and charge:

$$
\begin{aligned}
& N=\left|\begin{array}{ccccccc}
0 & 0 & 0 & 0 & 0 & 0 & \ldots \\
0 & 1 & 0 & 0 & 0 & 0 & \ldots \\
0 & 0 & 1 & 0 & 0 & 0 & \ldots \\
0 & 0 & 0 & 2 & 0 & 0 & \ldots \\
0 & 0 & 0 & 0 & 2 & 0 & \ldots \\
0 & 0 & 0 & 0 & 0 & 2 & \ldots \\
\ldots & \ldots & \ldots & \ldots & \ldots & \ldots & \ldots
\end{array}\right|, \\
& Z=\left|\begin{array}{ccccccc}
0 & 0 & 0 & 0 & 0 & 0 & \ldots \\
0 & 1 & 0 & 0 & 0 & 0 & \ldots \\
0 & 0 & -1 & 0 & 0 & 0 & \ldots \\
0 & 0 & 0 & 2 & 0 & 0 & \ldots \\
0 & 0 & 0 & 0 & 0 & 0 & \ldots \\
0 & 0 & 0 & 0 & 0 & -2 & \ldots \\
\ldots & \ldots & \ldots & \ldots & \ldots & \ldots & \ldots
\end{array}\right| .
\end{aligned}
$$

Furthermore, the particular action of $P_{0}$ on the states with given $N, Z$ is considered, as well as the possible transitions between states with fixed $N, Z$.

The last important thing present in the first part of the Majorana theory is the transformation from the basis where the states of the system were labelled according to $N, Z$ to the basis characterized by the operators:

$$
\frac{N+Z}{2}=L, \quad \frac{N-Z}{2}=M
$$

$(N=L+M, Z=L-M)$. The eigenvalues of both these operators were $0,1,2, \ldots$ and, as explicitly reported by Majorana, $M$ numbered the particles with negative charge while $L$ those with positive charge (that is, what Dirac in 1931 named "antielectrons"). The rewriting of the operators $Q_{0}, P_{0}, Q_{1}, P_{1}$ defined in the basis $N, Z$ in terms of $Q_{L}, P_{L}, Q_{M}, P_{M}$ defined in the basis $L, M$ then followed. Through these operators, $L$ and $M$ were written as:

$$
L=\frac{1}{2} P_{L}^{2}+\frac{1}{2} Q_{L}^{2}-\frac{1}{2}, \quad M=\frac{1}{2} P_{M}^{2}+\frac{1}{2} Q_{M}^{2}-\frac{1}{2} .
$$


The discussion of this first part ended with the pointing out of the particular case corresponding, in Majorana's own words, to the "classical theory", that is without what we name "anti-particles". This was achieved, for example, by assuming $P_{M}=$ $\psi_{M}=0$.

2.2. Majorana theory - second part. In the original manuscript, what just reported appears to have been considered a "preliminary" step, in Majorana's view, rather than a part of a complete theory. In fact at this point the author re-starts his calculation from a variational principle similar to that in Eq. (11), with the addition of the proper kinetic terms for the electromagnetic field:

$$
\begin{gathered}
\delta \int\left\{\frac { h ^ { 2 } } { 8 \pi ^ { 2 } m } \left[\frac{1}{c^{2}}\left(\frac{\partial}{\partial t}+\frac{2 \pi i}{h} e \varphi\right) \bar{\psi}\left(\frac{\partial}{\partial t}-\frac{2 \pi i}{h} e \varphi\right) \psi\right.\right. \\
\left.-\left(\boldsymbol{\nabla}-\frac{2 \pi i}{h c} e \boldsymbol{A}\right) \bar{\psi} \cdot\left(\boldsymbol{\nabla}+\frac{2 \pi i}{h c} e \boldsymbol{A}\right) \psi\right]-\frac{1}{2} m c^{2} \bar{\psi} \psi \\
\left.+\frac{1}{8 \pi}\left(\left|\frac{1}{c} \frac{\partial \boldsymbol{A}}{\partial t}+\boldsymbol{\nabla} \varphi\right|^{2}-|\boldsymbol{\nabla} \times \boldsymbol{A}|^{2}\right)\right\} \mathrm{d} \tau=0 .
\end{gathered}
$$

The variations taken with respect to $\psi, \bar{\psi}$ give, obviously, equations identical to those in Eqs. $(12,13)$, but now they were written in the following "mixed" form, where all the four fields $\psi, \bar{\psi}, P, \bar{P}$ appear:

$$
\begin{aligned}
& \left(\frac{\partial}{\partial t}-\frac{2 \pi i}{h} e \varphi\right) \bar{P}=-\frac{1}{2} m c^{2} \psi+\frac{h^{2}}{8 \pi^{2} m}\left|\nabla+\frac{2 \pi i}{h c} e \boldsymbol{A}\right|^{2} \psi, \\
& \left(\frac{\partial}{\partial t}+\frac{2 \pi i}{h} e \varphi\right) P=-\frac{1}{2} m c^{2} \bar{\psi}+\frac{h^{2}}{8 \pi^{2} m}\left|\nabla-\frac{2 \pi i}{h c} e \boldsymbol{A}\right|^{2} \bar{\psi} \\
& \left(\frac{\partial}{\partial t}+\frac{2 \pi i}{h} e \varphi\right) \bar{\psi}=\frac{8 \pi^{2} m c^{2}}{h^{2}} P, \\
& \left(\frac{\partial}{\partial t}-\frac{2 \pi i}{h} e \varphi\right) \psi=\frac{8 \pi^{2} m c^{2}}{h^{2}} \bar{P} .
\end{aligned}
$$

With the introduction of the quantities:

$$
\begin{aligned}
\rho & =\frac{h e}{4 \pi i m c^{2}}\left[\bar{\psi}\left(\frac{\partial}{\partial t}-\frac{2 \pi i}{h c} e \varphi\right) \psi-\psi\left(\frac{\partial}{\partial t}+\frac{2 \pi i}{h c} e \varphi\right) \bar{\psi}\right] \\
& =\frac{2 \pi i}{h} e(\psi P-\overline{\psi P}) \\
\boldsymbol{I} & =-\frac{h e}{4 \pi i m c}\left[\bar{\psi}\left(\boldsymbol{\nabla}+\frac{2 \pi i}{h c} e \boldsymbol{A}\right) \psi-\psi\left(\boldsymbol{\nabla}-\frac{2 \pi i}{h c} e \boldsymbol{A}\right) \bar{\psi}\right] \\
& =-\frac{h e}{4 \pi i m c}(\bar{\psi} \boldsymbol{\nabla} \psi-\psi \boldsymbol{\nabla} \bar{\psi})-\frac{e^{2}}{m c^{2}} \bar{\psi} \psi \boldsymbol{A} .
\end{aligned}
$$


S. ESPOSITO

The dynamical equations obtained by Eq. (37) with variations with respect to $\varphi$ and $\boldsymbol{A}$ are. respectively:

$$
\begin{aligned}
& \frac{1}{4 \pi} \nabla \cdot \boldsymbol{E}-\rho=0, \\
& \frac{1}{4 \pi c} \frac{\partial \boldsymbol{E}}{\partial t}-\frac{1}{4 \pi} \nabla \times \boldsymbol{H}+\boldsymbol{I}=0,
\end{aligned}
$$

that is the usual Maxwell equations with sources $(\boldsymbol{E}, \boldsymbol{H}$ are the electric and magnetic fields, respectively).

Some calculations were, at this stage, present in the manuscript aimed at realizing the (now) well-known difficulty, in a canonical theory, with the search for a field canonically conjugates to $\varphi$ (for $\boldsymbol{A}$ it is just $-\boldsymbol{E} / 4 \pi c$ ). Such calculations end with the "obvious" choice $\varphi=0$. Thus, summing up, the degrees of freedom of the system considered are described, in the theory elaborated by Majorana, by the (independent) fields:

$$
\psi, \bar{\psi}, \quad \boldsymbol{A},
$$

and their canonical conjugates:

$$
P=\frac{h^{2}}{8 \pi^{2} m c^{2}} \frac{\partial \psi}{\partial t}, \quad \bar{P}=\frac{h^{2}}{8 \pi^{2} m c^{2}} \frac{\partial \bar{\psi}}{\partial t}, \quad-\frac{\boldsymbol{E}}{4 \pi c}=\frac{1}{4 \pi c^{2}} \frac{\partial \boldsymbol{A}}{\partial t},
$$

while the Hamiltonian of the system is:

$$
\begin{aligned}
H= & \int\left[\frac{8 \pi^{2} m c^{2}}{h^{2}} \bar{P} P+\frac{1}{2} m c^{2} \bar{\psi} \psi+\frac{h^{2}}{8 \pi^{2} m} \nabla \bar{\psi} \cdot \boldsymbol{\nabla} \psi\right. \\
& \left.+\frac{h e}{4 \pi i m c} \boldsymbol{A} \cdot(\bar{\psi} \boldsymbol{\nabla} \psi-\psi \boldsymbol{\nabla} \bar{\psi})+\frac{c^{2}}{2 m c^{2}}|\boldsymbol{A}|^{2} \bar{\psi} \psi+\frac{1}{8 \pi}\left(E^{2}+H^{2}\right)\right] \mathrm{d} V .
\end{aligned}
$$

Such fields were, then, expanded in terms of three sets of plane waves (one set of scalar and two of vector quantities) as follows. Let us consider two sets of vector functions $\boldsymbol{f}_{\lambda}, \boldsymbol{f}_{k}^{\prime}$ labelled by the indices $\lambda, k$ :

$$
\begin{gathered}
\nabla^{2} \boldsymbol{f}_{\lambda}+\lambda^{2} \boldsymbol{f}_{\lambda}=0, \quad \nabla^{2} \boldsymbol{f}_{k}^{\prime}+k^{2} \boldsymbol{f}_{k}^{\prime}=0, \\
\int \boldsymbol{f}_{\lambda} \cdot \boldsymbol{f}_{\lambda^{\prime}} \mathrm{d} V=\delta_{\lambda \lambda^{\prime}}, \quad \int \boldsymbol{f}_{k}^{\prime} \cdot \boldsymbol{f}_{k^{\prime}}^{\prime} \mathrm{d} V=\delta_{k k^{\prime}}, \quad \int \boldsymbol{f}_{\lambda} \cdot \boldsymbol{f}_{k}^{\prime} \mathrm{d} V=0 .
\end{gathered}
$$

By imposing the constraint $\boldsymbol{\nabla} \times \boldsymbol{f}_{\lambda}=0$, the quantities $\boldsymbol{f}_{\lambda}$ may be written in terms of the gradient of some functions, $f_{\lambda}=\nabla u_{\lambda} / \lambda$, where

$$
\nabla^{2} u_{\lambda}+\lambda^{2} u_{\lambda}=0, \quad \int u_{\lambda} \cdot u_{\lambda^{\prime}} \mathrm{d} V=\int \boldsymbol{f}_{\lambda} \cdot \boldsymbol{f}_{\lambda^{\prime}} \mathrm{d} V=\delta_{\lambda \lambda^{\prime}} .
$$

In terms of these functions, the fields $\psi, P$ were written as:

$$
\begin{aligned}
& \psi=\frac{1}{\sqrt{2}} \sum_{\lambda} \sqrt{\frac{m c}{\sqrt{m^{2} c^{2}+\lambda^{2} h^{2} / 4 \pi^{2}}}}\left[q_{\lambda}+q_{\lambda}^{\prime}+i\left(p_{\lambda}-p_{\lambda}^{\prime}\right)\right] u_{\lambda}, \\
& P=\frac{h}{4 \pi \sqrt{2}} \sum_{\lambda} \sqrt{\frac{\sqrt{m^{2} c^{2}+\lambda^{2} h^{2} / 4 \pi^{2}}}{m c}}\left[p_{\lambda}+p_{\lambda}^{\prime}+i\left(q_{\lambda}-q_{\lambda}^{\prime}\right)\right] u_{\lambda},
\end{aligned}
$$


and similarly for $\bar{\psi}, \bar{P}$, where the four operators $q_{\lambda}, q_{\lambda}^{\prime}, p_{\lambda}, p_{\lambda}^{\prime}$ satisfy the commutation relations:

$$
\begin{gathered}
p_{\lambda} q_{\lambda}-q_{\lambda} p_{\lambda}=\frac{1}{i} \quad p_{\lambda}^{\prime} q_{\lambda}^{\prime}-q_{\lambda}^{\prime} p_{\lambda}^{\prime}=\frac{1}{i} \\
p_{\lambda} q_{\lambda}^{\prime}-q_{\lambda}^{\prime} p_{\lambda}=p_{\lambda}^{\prime} q_{\lambda}-q_{\lambda} p_{\lambda}^{\prime}=p_{\lambda} p_{\lambda}^{\prime}-p_{\lambda}^{\prime} p_{\lambda}=q_{\lambda} q_{\lambda}^{\prime}-q_{\lambda}^{\prime} q_{\lambda}=0 .
\end{gathered}
$$

For the electromagnetic field Majorana instead obtained:

$$
\begin{aligned}
\boldsymbol{A} & =\sum_{k} \sqrt{\frac{2 h c}{k}} Q_{k} \boldsymbol{f}_{k}^{\prime}+\sum_{\lambda} \frac{h c}{\sqrt{\pi}} P_{\lambda} \boldsymbol{f}_{\lambda}, \\
-\boldsymbol{E} & =\sum_{k} \sqrt{2 h c k} P_{k} \boldsymbol{f}_{k}^{\prime}-\sum_{\lambda} \sqrt{4 \pi} Q_{\lambda} \boldsymbol{f}_{\lambda},
\end{aligned}
$$

with

$$
P_{k} Q_{k}-Q_{k} P_{k}=\frac{1}{i}, \quad P_{\lambda} Q_{\lambda}-Q_{\lambda} P_{\lambda}=\frac{1}{i},
$$

the other commutators vanishing. ${ }^{4}$ The total charge of the system is given by

$$
-Z e=\int \rho \mathrm{d} V=\frac{2 \pi i}{h} e \int(\psi P-\overline{\psi P}) \mathrm{d} V,
$$

where

$$
\begin{aligned}
Z & =\sum_{\lambda}\left[\left(\frac{1}{2} p_{\lambda}^{2}+\frac{1}{2} q_{\lambda}^{2}-\frac{1}{2}\right)-\left(\frac{1}{2} p_{\lambda}^{\prime 2}+\frac{1}{2} q_{\lambda}^{\prime 2}-\frac{1}{2}\right)\right] \\
& =\sum_{\lambda}\left(N_{\lambda}-N_{\lambda}^{\prime}\right)=\sum_{\lambda} Z_{\lambda},
\end{aligned}
$$

with obvious meaning of the number operators $N_{\lambda}$ and $N_{\lambda}^{\prime}$. The number of the quanta of the electromagnetic field (photons) with frequency $\nu_{k}=c k / 2 \pi$ is instead given by:

$$
N_{k}=\frac{1}{2}\left(P_{k}^{2}+Q_{k}^{2}\right)-\frac{1}{2}
$$

The Hamiltonian of the complete system was written as

$$
H=H_{M}+H_{R}
$$

where

$$
H_{R}=\sum_{k} N h \nu_{k}+\sum_{\lambda} \frac{1}{2} Q_{\lambda}^{2}+\text { zero point energy }
$$

is the term describing radiation, and $H_{M}=H_{M}^{0}+H_{M}^{1}$ with

$$
H_{M}^{0}=\sum_{\lambda}\left(N_{\lambda}+N_{\lambda}^{\prime}\right) c \sqrt{m^{2} c^{2}+\lambda^{2} \frac{h^{2}}{4 \pi^{2}}}+\text { zero point energy }
$$

the free (matter) particle hamiltonian term, and $H_{M}^{1}$ describes the interaction among particles and between particles and light quanta.

Although an explicitly written physical interpretation of the formalism developed was not reported in the manuscript by Majorana (compare with what briefly discussed above in Sect. 1.4), it seems very clear that an independent (and, to some

\footnotetext{
${ }^{4}$ Formally, even for the electromagnetic field Majorana considered four operators $Q_{k}, Q_{\lambda}, P_{k}, P_{\lambda}$ instead of only two (for example $Q, P_{k}$ ); but, as we will see below, the number of free photons was given in terms of only $Q, P_{k}$.
} 
extent, different) formulation of the Pauli-Weisskopf theory was just formulated by the Italian physicist.

The original manuscript proceeded with some other calculations; here we point out only the following interesting things.

A transformation for the operators $q_{\lambda}, q_{\lambda}^{\prime}, p_{\lambda}, p_{\lambda}^{\prime}$ was introduced (apparently) in order to simplify the expressions in Eqs. (46):

$$
\begin{array}{ll}
a_{\lambda}=\frac{1}{\sqrt{2}}\left(q_{\lambda}+i p_{\lambda}\right), & b_{\lambda}=\frac{1}{\sqrt{2}}\left(q_{\lambda}^{\prime}+i p_{\lambda}^{\prime}\right), \\
\bar{a}_{\lambda}=\frac{1}{\sqrt{2}}\left(q_{\lambda}-i p_{\lambda}\right), & \bar{b}_{\lambda}=\frac{1}{\sqrt{2}}\left(q_{\lambda}^{\prime}-i p_{\lambda}^{\prime}\right) .
\end{array}
$$

The interesting point here is that the relations corresponding to Eqs. (47),

$$
\begin{gathered}
{\left[a_{\lambda}, \bar{a}_{\mu}\right]-\left[\bar{b}_{\lambda}, b_{\mu}\right]=2 \delta_{\lambda \mu}, \quad\left[\bar{a}_{\lambda}, a_{\mu}\right]-\left[b_{\lambda}, \bar{b}_{\mu}\right]=-2 \delta_{\lambda \mu},} \\
{[a, a]=[b, b]=[a, b]=[b, a]=0,}
\end{gathered}
$$

were written with the explicit notation

$$
[x, y]=x y \mp y x
$$

where the upper/lower sign referred to Bose-Einstein/Fermi-Dirac particles. Evidently Majorana was well conscious that the fundamentals of his theory applied to bosons as well as to fermions.

Other lengthy calculations were carried out in the original manuscript aimed at the quantization of the free radiation field in a different basis of plane waves (what we may call an helicity basis). Explicit expressions for the matrix elements of the operators appearing in the $\boldsymbol{A}, \boldsymbol{E}$ fields, the energy density $\left(\boldsymbol{E}^{2}+\boldsymbol{H}^{2}\right) / 8 \pi$ and the angular momentum density $(\boldsymbol{E} \times \boldsymbol{H}) / 4 \pi$ were reported (we address the interested reader to Sect.s 2.9-2.11 of Ref. [26]).

2.3. Possible application to the nuclear theory. An interesting application of what elaborated by Majorana on quantum electrodynamics was seemingly performed by him about the nuclear constitution. To put this into a context, first of all we take into account of a letter written by Majorana himself to his friend and colleague Giovanni Gentile Jr on December 22, 1929:

[...] I have read the article by Gamov that you pointed out to me; it seems to me that it gives really a good idea of what are the first stirrings of the rising theory of nuclei. This theory, however, has apparently no probability to reach its maturity unless we graft it onto the stump of quantum electrodynamics, that, in its turn, still gives its most pitful cries (read e.g. an article by Landau in the November issue of "Physikalische", if I am right).

In other words, it seems to me that the problem of the aggregation of protons and electrons in nuclei cannot have solutions, though approximate, until the problem of the constitution of protons and electrons themselves has been solved. This is for a very simple reason: the size of complex nuclei, as resulting from the Gamov theories, is of the same order of that of electrons (obviously, this is evaluated on classical grounds. Quantum mechanics has not brought, and cannot brought by itself, any light on this 


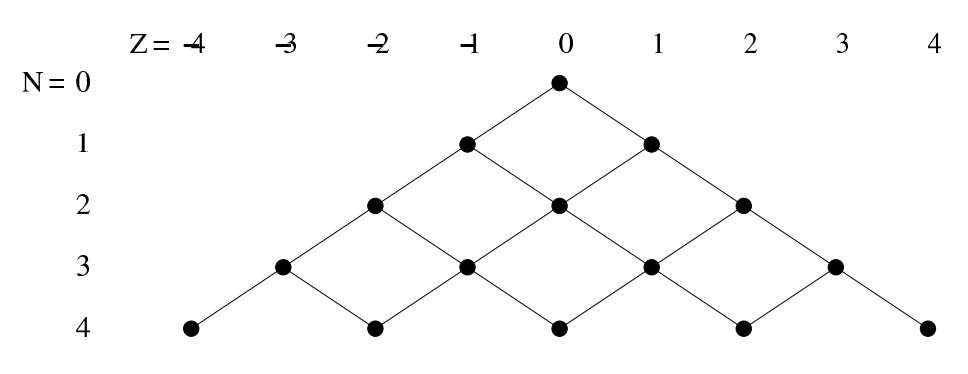

Figure 1. The building of nuclear states labelled by the total number of particles $N$ and the net charge $Z$.

question, since no relation between $e, h, m$ can hold due to dimensional reasons). Although such statements are largely vague, we can expect that something is hidden in them [...]. I am thus lead to believe that protons and electrons interpenetrate into nuclei in a sense different from that of wave mechanics, i.e. in a sense that is not liable to statistical interpretation [...]. [30]

At the time, in fact, it was commonly accepted that a nucleus $(Z, A)$ was composed of $A$ protons and $A-Z$ electrons [31], but the observations of the rotational Raman spectrum of gaseous nitrogen by Rasetti [28], interpreted ${ }^{5}$ by Walter Heitler and Gerhard Herzberg [32], opened a deep crisis in the current model of nucleus, which was correctly solved only with the discovery of the neutron in 1932. The mention above by Majorana was, then, nothing but the Heitler-Herzberg conclusion that (according to the current nuclear model) nuclear constituents "loose" their spin and the right to determine the statistics of the nucleus. ${ }^{6}$

Another trace of the interest of Majorana on such topics, related to the work by Rasetti on the spectra of diatomic molecules, was left in his Quaderni and, in particular, on his Quaderno No. 2, where several studies on diatomic molecules appear along with the calculations on quantum electrodynamics reported above.

Here, however, we want to focus on a possible application of the Majorana theory in Sect. 2.3 to the nucleus, which again was given in Quaderno No. 2, just mixed up with the developments of that theory, apparently according to Majorana's view of "grafting the theory of nuclei onto the stumps of quantum electrodynamics".

The two different kinds of scalar particles (endowed with positive and negative charge, respectively), described by the complex scalar field $\psi$ and its conjugate $P$ (together with $\bar{\psi}, \bar{P}$ are likely assumed to be the two nuclear constituents. The net charge was then, as usual, denoted by $Z$, while the total number of the constituents was $N$ (instead of $A$ ). Thus, in the present case, the number of protons

\footnotetext{
${ }^{5}$ The nucleus of nitrogen was assumed to be composed of 14 protons and 7 electrons, that is an odd total number of fermions, but on the contrary to expectations it was observed to follow the Bose-Einstein statistics, rather than the Fermi-Dirac one.

${ }^{6}$ It is right and proper to point out that the Heitler-Herzberg analysis of experimental data lead (correctly) to that conclusion only for electrons but, evidently, such a dissymmetry among the nuclear constituents (protons and electrons) sounded strange to Majorana, as well as to many other physicists.
} 


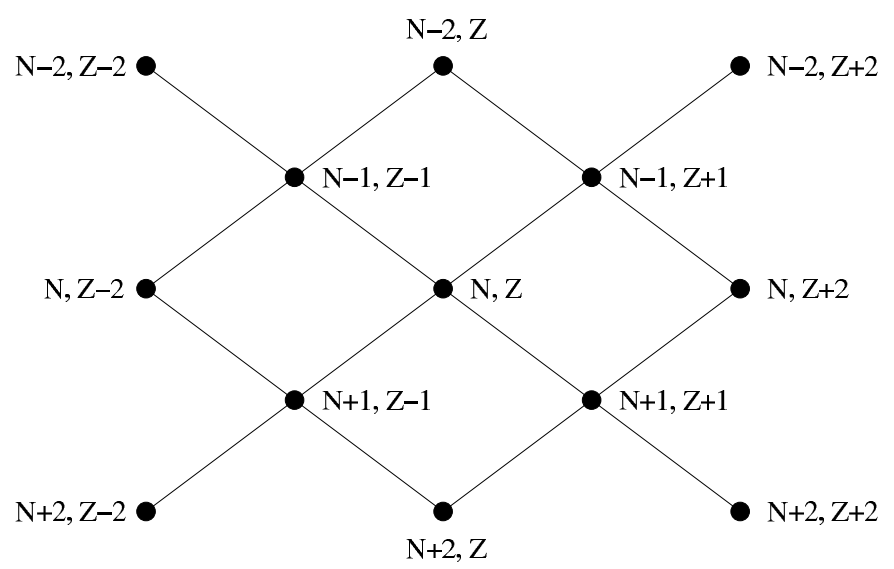

Figure 2. Possible transitions between nuclei with given $N, Z$.

and electrons would be $L$ and $M$, respectively (see Eq. (35)). ${ }^{7}$ It is not surprising such an association in the theory elaborated, given the large difference in mass between protons and electrons. In fact, it should be remembered that at the time when Majorana performed these studies, the Dirac theory of the electron still interpreted the "holes" of quantum electrodynamics in terms of protons (rather than antielectrons), despite its possible problem [15] [16].

Assuming such an interpretation, Majorana then built up a mass-charge $(N, Z)$ diagram for the possible nuclear states (see Figure 1) and, more important, a scheme for the possible transitions between nuclei of different $N, Z$ allowed in his theory (see Figure 2). For example, in this scheme the $\beta$-decay of a nucleus is described by a transition from $N, Z$ to $N-1, Z+1$. Majorana also calculated several matrix elements for these transitions, as already mentioned in Sect. 2.1, but this application was soon abandoned, probably due to the realization of the wrong predictions of the theory both on the compositions of nuclear states and on the transitions between them. Thus, differently from the elaboration of a theory for scalar electrodynamics, such an application remains just as an interesting historical curiosity.

\section{Conclusions}

The path to a complete and consistent formulation of a quantum electrodynamics, as recalled in Sect. 1, has undoubtedly one of its milestones in the development of the Pauli-Weisskopf theory for a scalar field. As seen, this is not mainly related to its possible direct applications, although the appearance of the Pauli-Weisskopf paper is quite rightly considered as the beginning of meson theory, but rather to the fact that it showed unambiguously that the marriage between quantum mechanics and special relativity did not necessarily require a spin $1 / 2$ for the correct interpretation of the formalism, as erroneously believed. According to Weisskopf himself, it was a "tremendous fun in working out something that, at that time, was quite unexpected:

\footnotetext{
${ }^{7}$ This notation is similar, but not identical to that used by Fermi [33], who denoted the atomic weight by $M$.
} 
that one can get pair creation and pair annihilation without a Dirac equation, also for particles without spin" [34].

In this respect, it is particularly relevant and interesting, from an historical point of view, the earlier formulation of such a theory by Majorana. This is not for a mere and quite fruitless priority issue, but rather for the discussion and consideration of the fundamental questions behind that theory as early as 1929-1930.

However, as should be clear from what reported in this paper, the Majorana theory presents also interesting theoretical peculiarities with respect to the PauliWeisskopf theory even for nowadays research, such as the use of general sets of plane waves in the expansion of the field variables or the adoption of four $\left(q_{\lambda}, q_{\lambda}^{\prime}, p_{\lambda}, p_{\lambda}^{\prime}\right.$, for matter particles) plus four $\left(Q_{k}, P_{k}, Q_{\lambda}, P_{\lambda}\right.$, for photons) instead of four plus two operators describing the quanta of the appropriate fields.

We then expect that further progress in these studies may certainly shed some new light on several important topics of present-day physics.

Acknowledgments. Interesting and valuable discussions with Erasmo Recami and Alberto De Gregorio are here kindly acknowledged.

\section{REFERENCES}

[1] E. Fermi, Rev. Mod. Phys. 4 (1932) 87, reproduced in Ref. [2].

[2] E. Fermi, Collected Papers, edited by E. Amaldi et al., University of Chicago Press - Accademia Nazionale dei Lincei, Chicago - Rome, 1962-1965, 2 volumes (1962).

[3] M. Born, W. Heisenberg and P. Jordan, Z. Phys. 35 (1926) 557.

[4] P.A.M. Dirac, Proc. Roy. Soc. London A 114 (1927) 243.

[5] P.A.M. Dirac, Proc. Roy. Soc. London A 114 (1927) 710.

[6] E. Fermi, Rend. Acc. Lincei 10 (1929) 72, reproduced in Ref. [2].

[7] P. Jordan and O. Klein, Z. Phys. 45 (1927) 755.

[8] P. Jordan and E. Wigner, Z. Phys. 47 (1928) 631.

[9] P. Jordan and W. Pauli, Z. Phys. 47 (1928) 151.

[10] W. Heisenberg and W. Pauli, Z. Phys. 56 (1929) 1.

[11] W. Heisenberg and W. Pauli, Z. Phys. 59 (1930) 168.

[12] E. Fermi, Rend. Acc. Lincei 9 (1929) 881, reproduced in Ref. [2].

[13] E. Fermi, Rend. Acc. Lincei 12 (1930) 431, reproduced in Ref. [2].

[14] P.A.M. Dirac, Proc. Roy. Soc. London A 117 (1928) 610, ibidem A 118 (1928) 351.

[15] H. Weyl, Z. Phys. 56 (1929) 330.

[16] P.A.M. Dirac, Proc. Roy. Soc. London A 126 (1930) 360.

[17] H. Weyl, Gruppentheorie und Quantenmechanik (Hirzel, Leipzig, 1931, second edition).

[18] P.A.M. Dirac, Proc. Roy. Soc. London A 133 (1931) 60.

[19] C.D. Anderson, Science 76 (1932) 238; Phys. Rev. 43 (1932) 491

[20] P.M.S. Blackett and G.P.S. Occhialini, Proc. Roy. Soc. London A 139 (1933) 699.

[21] W. Pauli, Wissenschaften Briefwechsel mit Bohr, Einstein, Heisenberg, U.A. II: 1930-1939 (Springer, Berlin, 1985).

[22] W. Pauli and V. Weiiskopf, Helv. Phys. Acta 7 (1934) 709.

[23] A.I. Miller, Early Quantum Electrodynamics (Cambridge University Press, Cambridge, 1994).

[24] See the comment by E. Amaldi in Ref. [2], Volume I, p. 305.

[25] S. Esposito, E. Majorana jr, A. van der Merwe and E. Recami, Ettore Majorana: Notes on Theoretical Physics (Kluwer/Springer, New York, 2003).

[26] S. Esposito, E. Recami, A. van der Merwe and R. Battiston, Ettore Majorana: Research Notes on Theoretical Physics, Springer, to appear.

[27] E. Recami, Il caso Majorana. Epistolario, documenti, testimonianze (Oscar Mondadori, Milan, 1991, second edition); idem (Di Renzo, Rome, 2002, fourth revised and enlarged edition).

[28] F. Rasetti, Nature 123 (1929) 205; Proc. Nat. Acad. Sci. 15 (1929) 234, 515.

[29] G.C. Wick, Atti Acc. Sci. Torino, Suppl. 2 Vol. 115 (1981) 13.

[30] This letter (in Italian) is reported in Ref. [27]. 
[31] A. De Gregorio, "Il "protone neutro". Ovvero della laboriosa esclusione degli elettroni dal nucleo," arXiv:physics/0603261. See also R.H. Stuewer, "The Nuclear Electron Hypothesis," in W.A. Shea, ed., Otto Hahn and the Rise of Nuclear Physics (Reidel, Dordrecht, 1983), p. 19.

[32] W. Heitler and G. Herzberg, Naturwiss. 17 (1929) 673.

[33] E. Fermi in La Physique du noyau atomique, Comptes Rendus de la premiere section du Congrès International d'électricite (Gauthier-Villars, Paris, 1932); Ric. Sci. 3 (1932) 101, reproduced in Ref. [2].

[34] V.F. Weisskopf, talk given at the Erice Summer School in High-Energy Physics, Erice (Italy), 1971.

S. Esposito: Dipartimento di Scienze Fisiche, Università di Napoli "Federico II" \& I.N.F.N. Sezione di Napoli, Complesso Universitario di M. S. Angelo, Via Cinthia, 80126 NAPOLI (Salvatore.Esposito@na.infn.it) 